\title{
Correction to: Density, viscosity, surface tension, spectroscopic properties and computational chemistry of the 1, 4-butanediol + 1 , 3-propanediamine-based deep eutectic solvent
}

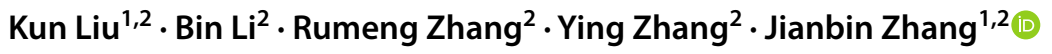

Published online: 18 November 2021

(c) Iranian Chemical Society 2021

\section{Correction to: Journal of the Iranian Chemical Society https://doi.org/10.1007/s13738-021-02371-0}

There was a mistake in the name in affiliation 2 which is corrected through this correction.

The original article can be found online at https://doi.org/10.1007/ s13738-021-02371-0.

Jianbin Zhang

tadzhang@pku.edu.cn

1 Hebei Provincial Key Lab of Green Chemical Technology and High Efficient Energy Saving, School of Chemical Engineering and Technology, Hebei University of Technology, Tianjin 300130, China

2 Inner Mongolia Engineering Research Center for $\mathrm{CO} 2$ Capture and Utilization, College of Chemical Engineering, Inner Mongolia University of Technology, Hohhot 010051, China 